Pure Appl. Chem., Vol. 72, Nos. 1-2, pp. 199-207, 2000.

(C) 2000 IUPAC

\title{
Distribution and shape of self-assembled InAs quantum dots grown on GaAs (001)*
}

\author{
K. Zhang ${ }^{1}$, J. Falta2,3, Th. Schmidt2,3, Ch. Heyn¹, G. Materlik², \\ and W. Hansen 1
}

${ }^{1}$ Institut für Angewandte Physik, Universität Hamburg, Jungiusstrasse 11, D-20355

Hamburg, Germany; ${ }^{2 H a m b u r g e r ~ S y n c h r o t r o n s t r a h l u n g s l a b o r ~ H A S Y L A B ~ a m ~}$

Deutschen Elektronen-Synchrotron DESY, Notkestrasse 85, D-22603 Hamburg,

Germany

\begin{abstract}
Grazing incidence small angle X-ray scattering (GISAXS) and atomic force microscopy (AFM) experiments are employed to study self-assembled InAs quantum dots (QDs) grown by molecular beam epitaxy (MBE) on GaAs (001). The GISAXS spectra show pronounced non-specular diffuse scattering satellite peaks with high diffraction orders up to \pm 3 along [110], [1-10], and [100] sample azimuthal orientations with respect to the incoming beam, indicating a lateral ordering of the InAs QDs. The correlation lengths of the lateral dot distribution are found to be identical along [110] and [1-10] but smaller along [100] direction. The ratio of the mean dot-dot distances along [100] and [1-10] azimuths is determined to be 1.13 , indicating the anisotropic ordering of QD distribution. Broad diffraction peaks are observed at larger scattering angles and associated to dot facet crystal truncation rods (CTR). We determine $\{111\}$-like facets along [110] and [1-10] sample azimuths, and $\{101\}$-like facets along [100] azimuth.
\end{abstract}

\section{INTRODUCTION}

It is well known that in the heteroepitaxy of materials with a large lattice mismatch to the substrate (e.g., $\mathrm{SiGe}$ on $\mathrm{Si}$ or InAs on GaAs) small droplets are formed in the so-called Stranski-Krastanow growth mode if a critical layer thickness of a wetting layer is exceeded [1]. Studies with atomic force microscopy (AFM) or transmission electron microscopy (TEM) reveal that the dots can be coherently strained, dislocation-free, and have in the case of InAs on GaAs diameters of a few $10 \mathrm{~nm}$ with a surprisingly narrow size distribution [2]. A few years ago the first experimental evidence for the existence of zerodimensional electronic states in self-assembled InAs quantum dots (QDs) was obtained with capacitance and far-infrared spectroscopy [3]. Since then, this type of QD has been studied very intensively by means of a wide spectrum of techniques in view of both fundamental aspects, as well as potential technological applications [4]. The atomic-like electron states have been probed by, e.g., photoluminescence [5,6], cathodoluminescence [7], capacitance measurements [8], and far-infrared spectroscopy $[9,10]$, as well as ballistic electron emission spectroscopy [11].

Great effort is also devoted to characterization, understanding, and improvement of the structural properties of such QD nanostructures, as reported in, e.g., [12-16]. The dot formation process is thought to be entirely driven by the strain relaxation induced by the large strain energy associated with the lattice mismatch of up to 7\%. In models for strain-induced self-assembling and ordering it is pointed out that vertical stacking of many layers of QDs, separated by thin spacer layers, may enhance the

*Pure Appl. Chem. 72, 1-331 (2000). An issue of reviews and research papers based on lectures presented at the $1^{\text {st }}$ IUPAC Workshop on Advanced Materials (WAM1), Hong Kong, July 1999, on the theme of nanostructured systems.

${ }^{3}$ Present address: Institut für Festkörperphysik, Universität Bremen, Postfach 330440, D-28334 Bremen, Germany 
lateral and vertical ordering as well as the size homogeneity [14]. Experimental studies qualitatively verify the theoretical predictions [12]. It has been shown experimentally that the strain field of a QD extends far into the underlying wetting layer and substrate [17,18]. This strain field surrounding each QD may induce dot-dot interaction that strongly influences the growth of neighboring dots and thus causes short-range ordering of neighboring dots. This scheme has been applied to interpret the vertical ordering in a multilayer of InAs-GaAs QDs [7,19]. Experimentally, high-resolution X-ray diffraction plays an important role in the study of the structure in the vertical stacking InAs QD sample [15]. However, as to a single dot layer structure, there is little experimental knowledge about the lateral ordering character of QDs, and X-ray diffraction measurements are difficult since the volume fraction of InAs dots is very small; hence the scattering intensity generated from InAs QD is very weak.

The shape of InAs QD is also debated intensively. For example, an accurate calculation of the electronic structure depends critically on the assumptions of the QD shape [20]. Square-based pyramids [21,22], lens shapes, and conical dot shapes [5] have been proposed, and complex numerical models are developed to predict the dot shape, e.g., an octagonal-based shape predicted by Moll et al. [23]. Various experimental techniques like atomic force microscopy (AFM) [24,25], reflection high-energy electron diffraction (RHEED) [26,27], scanning tunneling microscopy (STM) [28,29], and transmission electron microscopy (TEM) $[2,7,12]$ have been employed in order to determine the shape of the dots. However, the results of the above techniques so far do not provide a uniform picture for the shape of the QDs.

In order to study the distribution and shape of the uncapped InAs QD single layer experimentally, in the present work we perform novel high-resolution GISAXS experiments [30] employing synchrotron radiation. The grazing incidence of the high-flux synchrotron radiation beam probes the crystal surface up to a depth of only a few nanometers so that the volume fraction of InAs is strongly enhanced in comparison to the conventional scattering geometry. Furthermore, as compared to AFM measurements a relatively large area contributes coherently to the scattering so that the statistics of the measurements is improved. In addition, the GISAXS technique allows the determination of the facet families and the average facet sizes of the InAs QDs. Most importantly, it provides us an opportunity to investigate InAs QD layers buried beneath a GaAs cap layer that would obstruct AFM studies. Such studies are presently under way.

\section{SAMPLE GROWTH}

Two types of samples are prepared in a conventional MBE system (Riber 32) with base pressure of $1 \times 10^{-11} \mathrm{mbar}$. In situ RHEED was used to monitor the growth process. The InAs growth speed was $0.07 \mathrm{ML} / \mathrm{s}$ as determined from the RHEED oscillation frequency during GaAs and $\mathrm{Ga}_{0.9} \mathrm{In}_{0.1}$ As growth, and the arsenic flux corresponds to a flux gauge reading of $1 \times 10^{-6}$ mbar. After oxide desorption, a 200-nm-thick GaAs buffer was grown on (001) GaAs substrates at $600^{\circ} \mathrm{C}$. The RHEED pattern showed a $2 \times 4$ reconstruction with surface diffraction streaks. Afterwards, the substrate temperature was decreased down to $500{ }^{\circ} \mathrm{C}$, resulting in a change of the GaAs surface reconstruction to $\mathrm{c}(4 \times 4)$ with surface diffraction streaks (Fig. 1a). After the Indium shutter was opened, the reconstruction features instantaneously disappear, but still the RHEED patterns show a two-dimensional surface morphology (Fig. 1b). The transition from 2D to 3D InAs growth was found to be at 1.7 ML InAs deposited. At this point, the 2D diffraction spots disappear (Fig. 1c) before new 3D-type diffraction spots emerge. In Fig. 1d the diffraction pattern observed at 2.1 ML InAs coverage is displayed. It has been changed from 2D surface-related features to 3D bulk diffraction spots due to the transition of the growth mode from layer to island growth. It can be clearly seen that the 3D diffraction spots are decorated with additional weak intensity tails, so-called chevrons [27]. We note that besides the 3D diffraction spots there still exists a surface specular beam spot at which no chevron is attached. We terminate the InAs growth after 

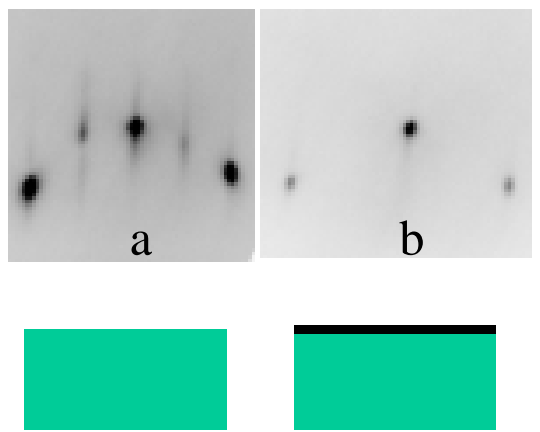
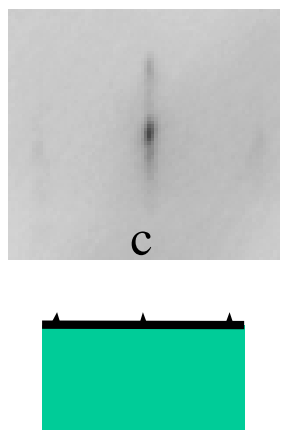
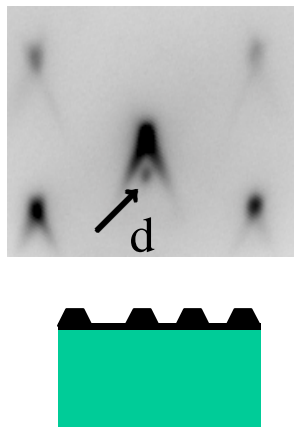
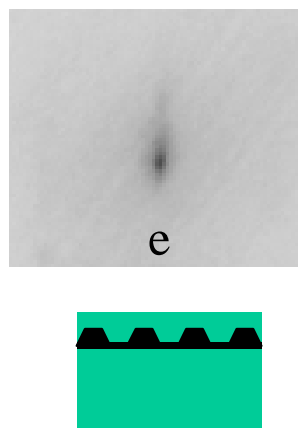

Fig. 1 In situ RHEED patterns observed during MBE growth of InAs on GaAs (001). (a) substrate at $500{ }^{\circ} \mathrm{C}$, (b) start of InAs growth, (c) transition to 3D growth, (d) 2.1 ML InAs deposited, and (e) after deposition of the GaAs cap. The arrow in Fig. 1d indicates the specular beam spot.

2.1 ML deposition. In the first type of samples the InAs dot layer was covered after $30 \mathrm{~s}$ delay time with a 5-nm-thick GaAs cap that was grown at the same substrate temperature as the InAs layer. The corresponding RHEED pattern is presented in Fig. 1e showing a surface specular beam spot only. From this we infer that the GaAs cap layer has considerable roughness [31]. The first type of sample was used to characterize the surface and interface structure by grazing incidence X-ray reflectivity, crystal truncation rod, as well as X-ray standing waves [31]. The second type of sample was grown very similarly except that the sample was rotated during InAs growth and no cap was deposited. While sample rotation prevents us from monitoring the InAs growth with RHEED, it will improve the homogeneity of the QD size and distribution on the wafer. The RHEED pattern before and after deposition of the InAs layer does not differ from the previous samples. In the following, we focus on our studies of the second type samples. They remained uncapped for our AFM measurements and in order to increase the intensity in our GISAXS experiments. As soon as the growth was finished, both types of samples were quenched by stopping the substrate heater.

\section{RESULTS AND DISCUSSIONS}

\section{AFM measurement of InAs QDs}

The topology of our InAs QDs was first investigated using a Topometrix-AFM with a cantilever tip of $10 \mathrm{~nm}$ diameter. The AFM measurement were taken with a scan range of $1 \times 1 \mu \mathrm{m}^{2}$. Fig. $2 \mathrm{a}$ shows a zoom of a typical AFM image. A quantitative analysis of the AFM image indicates that the dots exhibit a narrow size distribution. The average dot height and lateral size are determined to about $4 \pm 1 \mathrm{~nm}$ and $30 \pm 10 \mathrm{~nm}$ respectively. The dot density is about $5.0 \times 10^{10} \mathrm{~cm}^{-2}$. The lateral ordering of the dots can be evaluated by a one-dimensional Fourier transformation of the AFM image. In Fig. 2b the result for the [110] direction is presented. We find a weak satellite peak indicated by the arrow as in Fig. 2b. The existence of the satellite peak is a direct evidence for a lateral ordering of the InAs QDs. From the peak position, we estimate the mean dot-dot distance to be about $67 \pm 8 \mathrm{~nm}$ in the [110] direction. In [1-10] direction the satellite peaks are much weaker and hence the mean dot-dot distance can hardly be determined. We note that the above distance is a scale of the laterally periodic distribution or "most frequent" dot-dot separation, and it may differ from an average distance determined from the above-dot density assuming a perfect square lattice. The shape of the QD facets cannot be determined from our AFM measurements since the size of these is beyond our instrumental resolution. 


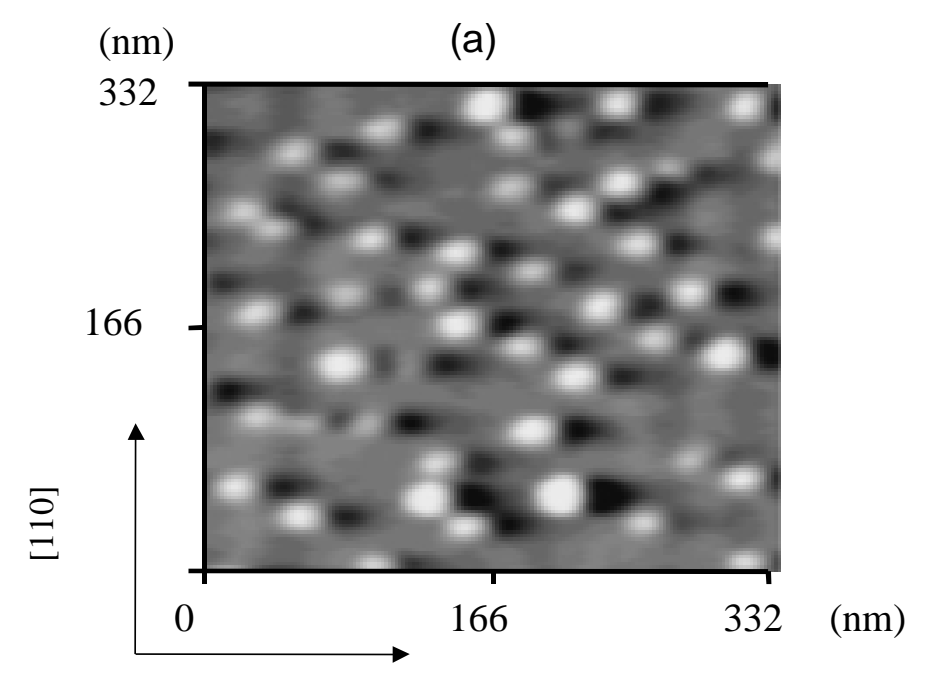

[1-10]

(b)

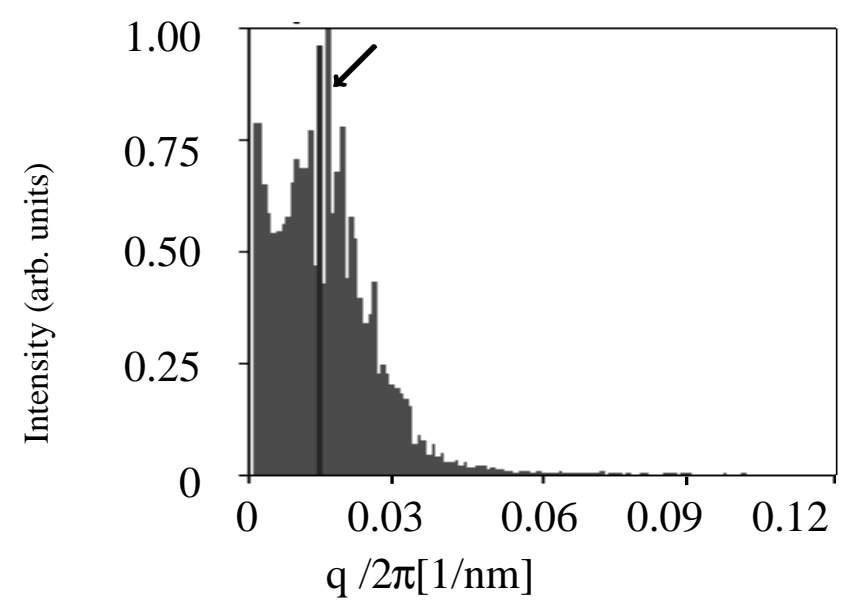

Fig. 2 AFM image of self-assembled InAs QDs. Left shading at an angle of $45^{\circ}$ to the horizontal direction is processed to the AFM image in order to enhance visibility (a). One-dimensional Fourier transformation of $1 \times 1 \mu \mathrm{m}^{2} \mathrm{AFM}$ image in [110] direction (b).

\section{GISAXS measurement}

GISAXS measurements were performed at the DESY undulator beamline BW1 at a wavelength of $1.17 \AA$. The GISAXS setup is shown schematically in Fig. 3. The incidence angle $\alpha_{i}$ relative to the sample surface is $0.18^{\circ}$, which is below the total external reflection angle of $0.20^{\circ}$. The illuminated area on the sample is $16 \times 2 \mathrm{~mm}^{2}$. At this incidence angle the penetration depth of the incoming beam is about $6 \mathrm{~nm}$. Therefore, the reflection intensity is enhanced and very sensitive to the top InAs QD surface layer. Profiles of the scattered intensity are collected by a position sensitive detector (PSD), which is mounted parallel to the sample surface at an exit angle of $\alpha_{\mathrm{f}}$. In order to enhance the scattering intensity relative to the very strong specular beam, the detector is slightly moved out of the specular beam 
$\left(\alpha_{\mathrm{f}}>\alpha_{\mathrm{i}}\right)$. Due to the size of the slits used in the experiments, the resolution parallel to PSD is limited to $2.1 \times 10^{-3} \AA^{-1}$. With this resolution a mean dot-dot distance of up to $300 \mathrm{~nm}$ can be determined.

\section{Mean dot-dot distance determination}

We have performed GISAXS measurements of self-assembled InAs QD for different sample azimuthal orientations $\Omega$. As sketched in Fig. 3, the azimuth is changed with an angular step of $5^{\circ}$ by rotation around the sample surface normal. We chose $-10^{\circ} \leq \Omega \leq 110^{\circ}$, where $\Omega=0$ is set at [110] azimuthal direction. We find nonspecular satellite peaks in all azimuths, although in some cases, the satellite peaks are weak. As an example, we present GISAXS satellite peak profiles in Fig. 4 for [1-10], [110], [100] azimuths. Satellite peaks located nearly symmetrically at both sides of the specular beam $\left(q_{x}=0 \AA^{-1}\right)$ are clearly resolved for these three azimuths. Most importantly, in our case they exhibit high orders of satellite peaks in above azimuths, i.e., $\pm 1, \pm 2, \pm 3$ orders. By comparison with reference samples which
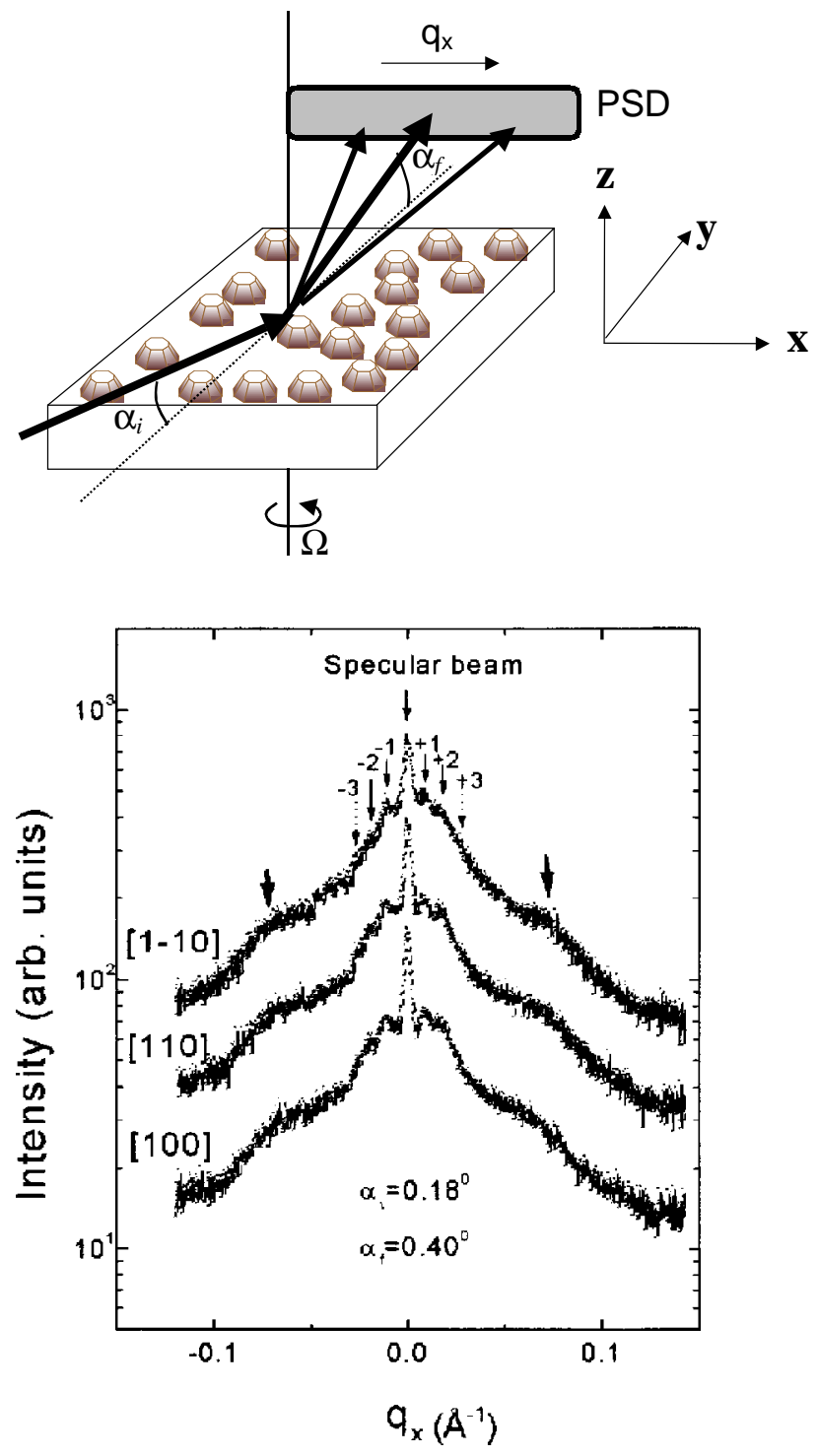

Fig. 3 Schematic illustration of GISAXS experimental setup. $\alpha_{i-}$ incidence angle, $\alpha_{f}$ _exit angle. The dotted line collimates to the incoming beam. $\Omega$ denotes the angle of sample rotation around the surface normal.
Fig. 4 The logarithmic GISAXS intensities along $\mathrm{q}_{\mathrm{x}}$ of self-assembled InAs QDs for different azimuthal sample orientations with respect to the incoming beam. For clarity, the upper curves are displaced by a magnitude relative to the lower one, respectively. The central peak at $\mathrm{q}_{\mathrm{x}}=0$ is from the specular beam intensity. The satellite peaks with higher orders are indicated by $\pm 1, \pm 2, \pm 3$. The additional broad maxima in GISAXS at $\mathrm{q}_{\mathrm{x}}= \pm 0.07 \AA^{-1}$ are indicated by thick arrows. The incidence angle $\mathrm{a}_{\mathrm{i}}$ equals to $0.18^{\circ}$, which is below the total external reflection angle. 
contain no QDs and show no satellite peaks, we can conclude that the satellite peaks are caused by diffuse scattering of the InAs QDs. The occurrence of well-resolved scattering peaks and their higher orders are a strong indication of ordering in the dot arrangement.

The mean dot-dot distance $<\mathrm{d}>$ is determined by the first satellite peak position with respect to the origin, i.e., $\langle d\rangle=2 \pi / \mathrm{q}_{\mathrm{x}}[30]$. In [110] and [1-10] azimuths we find the same value $\langle\mathrm{d}\rangle=64 \pm 2 \mathrm{~nm}$, whereas in [100] azimuth the data yield $\langle\mathrm{d}\rangle=72 \pm 2 \mathrm{~nm}$. We note that the value measured in $\langle 110\rangle$ azimuth compares well with the mean dot-dot distance determined from our AFM data. Comparing the values for the mean dot-dot distance found in our GISAXS experiments in $\langle 110\rangle$ and $\langle 100\rangle$ directions we determine a ratio $\mathrm{R}=\langle\mathrm{d}\rangle_{100} /\langle\mathrm{d}\rangle_{1-10} \cong 1.13$. This value indicates ordering since it is significantly larger than the value of $\mathrm{R}=1.0$ expected for an isotropic dot distribution. However, it is still smaller than $\mathrm{R}=1.41$ which would be expected for a perfect square lattice.

\section{Mean correlation length of the lateral dot distribution}

For an appropriate fit to the GISAXS intensity we use a sum of a Gaussian function for the specular beam and Lorentzian functions for the satellite peaks. From the fits, we can determine full width at half maximum (FWHM) values of the first order satellite peaks. The FWHM of the satellite peaks may either originate from a large-scale dot density inhomogeneity or from a finite correlation length of the short-range order. AFM measurements on different parts of our wafer show the dot density to be very homogeneous. We thus regard the FWHM values in our case mainly due to the local disorder among the nearest neighbors of each dot row in a certain azimuth as described by Schmidbauer et al. [30]. Then a correlation length of the dot distribution can be obtained that quantitatively reflects the degree of ordering in the lateral dot distribution. By an appropriate deconvolution of the FWHM of the satellite peaks with an instrumental resolution function, we obtain the standard deviation of the dot-dot distance along [110] and [1-10] and [100] azimuths [32]. Assuming short-range order type of the correlation function [30] this implies correlation lengths of $\mathrm{L}=102 \pm 5 \mathrm{~nm}$ along [110] and [1-10] azimuths and $90 \pm 5 \mathrm{~nm}$ along the [100] azimuth, respectively. This indicates that the ordering of the dot distribution along [110] and [1-10] is slightly higher than along [100] azimuth.

\section{Spatial distribution of integrated intensity of satellite peaks}

We determine the integrated intensities $\langle\Phi\rangle$ of the satellite peaks at different azimuths and present the values normalized to the corresponding specular beam intensities in Fig. 5. The integrated intensity

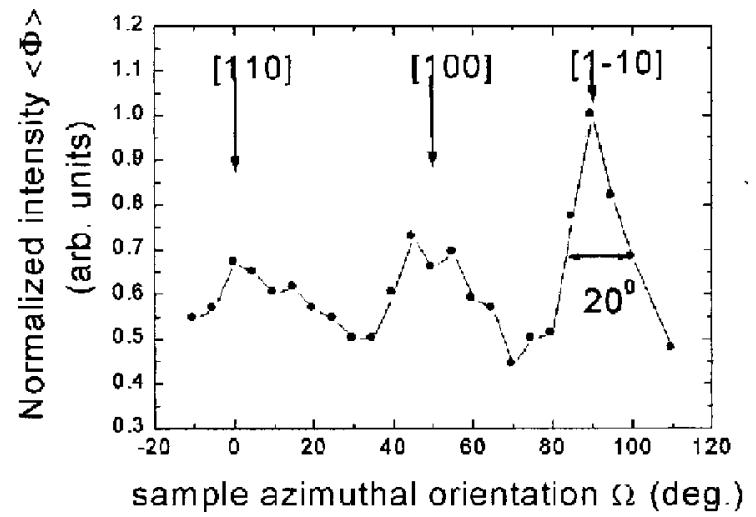

Fig. 5 Normalized integrated intensity $\langle\Phi\rangle$ distribution of the satellite peaks versus sample azimuthal orientations with respect to the incoming beam. [110] azimuth is set to $0^{\circ}$ of azimuthal orientation. For each azimuth the integrated intensity is normalized by the corresponding specular beam intensity. In the figure the $\langle\Phi\rangle$ value is presented with respect to the intensity observed in [1-10] azimuth. 


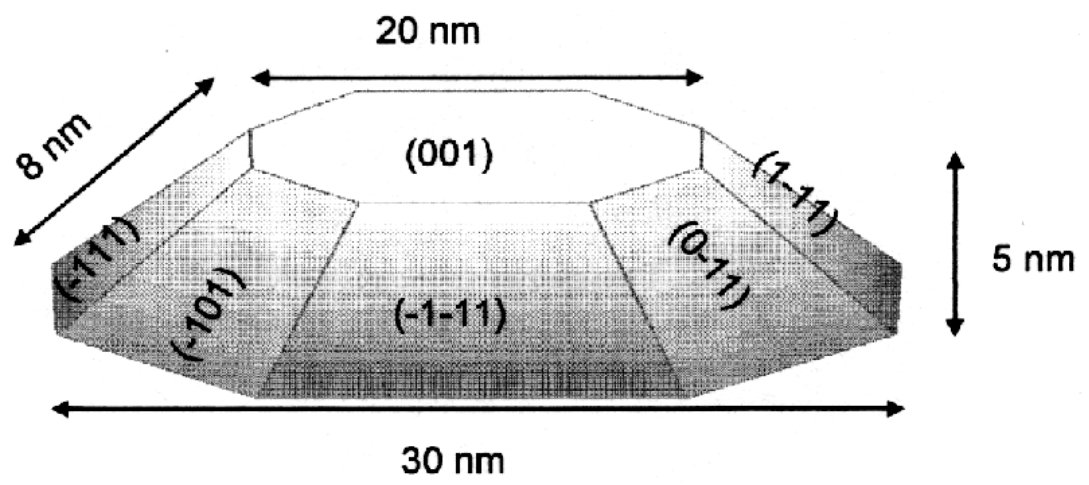

Fig. 6 The sketch of InAs QD shape as determined by the facet families and dot geometry. The lateral bottom size along [1-10] direction is estimated by AFM.

distribution has three maxima located at angles $0^{\circ}, 45^{\circ}$, and $90^{\circ}$, respectively. These reflect enhanced ordering of the lateral dot distribution in the corresponding azimuthal orientations, i.e., [110], [100] and [1-10]. The peak widths of the distribution in Fig. 5 reflect the deviation of the dot position from a straight row. For example, in [1-10] direction we find an azimuthal width of $20^{\circ}$ which is in close correspondence to the observation on $\mathrm{SiGe/Si} \mathrm{[30].} \mathrm{In} \mathrm{addition,} \mathrm{we} \mathrm{note} \mathrm{the} \mathrm{smaller} \mathrm{azimuthal} \mathrm{width}$ along [1-10] azimuth as compared to [110] azimuth in Fig. 5, suggesting the higher ordering along [1-10] azimuth than along [110] azimuth.

\section{$Q D$ facet shape determination}

Intriguingly, we find additional, broad maxima in the GISAXS intensity curves located at $\mathrm{q}_{\mathrm{x}}= \pm 0.07 \AA^{-1}$. They are highlighted by arrows in Fig. 4. Since the PSD is located at finite values of $\mathrm{q}_{\mathrm{z}}$ rather than $\mathrm{q}_{\mathrm{z}}=0$ in our experiment, the broad maxima may result from the CTR intensity of the InAs QD facets. In order to clarify this we have investigated the dependence of the facet CTR peak position in $\mathrm{q}_{\mathrm{x}}$ with different values of $\mathrm{q}_{\mathrm{z}}$ for azimuthal orientations of [110], [1-10], and [100], respectively. We find that the facet CTR is tilted with respect to the [001] surface normal by $54.1^{\circ}$ along [1-10] azimuth. This strongly suggests that the peaks are associated to CTR intensity of the $\{111\}$ facet family. The same facet family is also observed along [110] azimuth. Intriguingly, our investigation in [100] azimuth indicates that our dots are not only formed by $\{111\}$ facets but also contain facets of the $\{101\}$ family. Thus, the InAs QDs formed with these facets have an octagonal-based shape. We note that our observation partially corresponds with TEM investigations by Grundmann et al. [7] where other facets are inferred besides the facets of the $\{011\}$ family. Furthermore, the determination of $\{111\}$ and $\{101\}$ families of InAs QD facets in our case is also in good agreement with a finite element calculation for equilibrium surface state InAs QDs [23]. Other facets have been inferred from STM measurements $(\{113\},\{114\}$, and $\{215\}$ facet families [28,29]), from AFM data (\{410\} family [25]), and from RHEED patterns ( $\{113\}$ [26], $\{136\}$ facet families [27]). In our experiments we do not resolve indications for such facets. A detailed discussion of the facet CTR will be published in a forthcoming publication [32]. From the broadening of the facet CTR peaks an estimate can be given for the average facet size [30,33]. In our case we get a facet width of approximately $8 \pm 2 \mathrm{~nm}$. From this we calculate a dot height of about $5 \mathrm{~nm}$, which is in good agreement with our AFM data. Combining our results from AFM and GISAXS on the facet size and families, and the dot diameter and height, we suggest a dot shape as sketched in Fig. 6. The dots have facets of $\{111\}$ and $\{101\}$ families which are truncated by a (001) top surface facet.

(C) 2000 IUPAC, Pure and Applied Chemistry 72, 199-207 


\section{CONCLUSIONS}

We employ RHEED, AFM imaging, and synchrotron radiation experiments to study structural properties of self-assembled InAs QDs on (001) GaAs. In particular, here we employ GISAXS, which represents a powerful technique to obtain information on the geometry of the dot shape as well as the distribution within the plane of the wetting layer. Important structural parameters characterizing the lateral distribution and the shape of InAs QDs are derived in the present work. The shape of InAs QDs is determined as a truncated octagonal-based pyramid, which is in good agreement with the result of finite element calculation for the equilibrium surface state of InAs QDs [23]. Lateral ordering of the InAs QD distribution is found at least along $\langle 110\rangle$ and $\langle 100\rangle$ azimuths. For our sample we find a mean dot separation of $64 \mathrm{~nm}$ in $\langle 110\rangle$ directions. The azimuthal dependence of the satellite peak position of GISAXS points to an anisotropic dot distribution with a larger dot separation of $72 \mathrm{~nm}$ in $\langle 100\rangle$ direction. This may be attributed to strain field anisotropies. Further studies are needed to clarify this issue.

\section{ACKNOWLEDGMENTS}

The GISAXS experiments have been performed at the HASYLAB. We gratefully acknowledge financial support by the Graduiertenkolleg "Physik nanostrukturierter Festkörper", and also Dr. Joachim Wollschlaeger for helpful discussion.

\section{REFERENCES}

1. I. N. Stranski and L. von Krastanow. Akad. Wiss. Lit. Mainz Math.-Natur. Kl. IIb 146, 797 (1939).

2. D. Leonard, M. Krishnamurthy, C. M. Reaves, S. P. Denbaars, P. M. Petroff. Appl. Phys. Lett. 63, 3203 (1993).

3. H. Drexler, D. Leonard, W. Hansen, J. P. Kotthaus, P. M. Petroff. Phys. Rev. Lett. 73, 2252 (1994).

4. D. Bimberg, M. Grundmann, N. N. Ledentsov. Quantum Dot Heterostructures, John Wiley, UK (1998).

5. J.-Y. Marzin and G. Bastard. Solid State Commun. 92, 437 (1994).

6. K. H. Schmidt, G. Medeiros-Ribeiro, J. Garcia, P. M. Petroff. Appl. Phys. Lett. 70, 1727 (1997).

7. M. Grundmann, N. N. Ledentsov, R. Heitz, L. Eckey, J. Christen, J. Böhrer, D. Bimberg, S. S. Ruvimov, P. Werner, U. Richter, J. Heydenreich, V. M. Ustinov, A. Yu. Egorov, A. E. Zhukov, P. S. Kop'ev, Z. I. Alferov. Phys. Status Solidi B 188, 249 (1995).

8. B. T. Miller, W. Hansen, S. Manus, R. J. Luyken, A. Lorke, J. P. Kotthaus, S. Huang, G. MedeirosRibeiro, P. M. Petroff. Phys. Rev. B 56, 6764 (1997).

9. M. Fricke, A. Lorke, J.P. Kotthaus, G. Medeiros-Ribeiro, P. M. Petroff. Europhys. Lett. 36, 197 (1996).

10. S. Sauvage, P. Boucaud, F. H. Julien, J.-M. Gerard, J. Y. Marzin. J. Appl. Phys. 82, 3396 (1997).

11. M. E. Rubin, G. Medeiros-Ribeiro, J. J. O'Shea, M. A. Chin, E. Y. Lee, P. M. Petroff, V. Narayanamurti. Phys. Rev. Lett. 77, 5268 (1996).

12. Q. Xie, A. Madhukar, P. Chen, N. P. Kobayashi. Phys. Rev. Lett. 75, 2542 (1995).

13. V. Bressler-Hill, S. Varma, A. Lorke, B. Z. Nosho, P. M. Petroff, W. H. Weinberg. Phys. Rev. Lett. 74, 3209 (1995).

14. J. Tersoff, C. Teichert, M. G. Lagally. Phys. Rev. Lett. 76, 1675 (1996).

15. A. A. Darhuber, V. Holy, J. Stangl, G. Bauer, A. Krost, F. Heinrichsdorff, M. Grundmann, D. Bimberg, V. M. Ustinov, P. S. Kopev, A. O. Kosogov, P. Werner. Appl. Phys. Lett. 70, 955 
(1997); A. A. Darhuber, P. Schittenhelm, V. Holy, J. Stengl, G. Bauer, G. Abstreiter. Phys. Rev. B 55, 15652 (1997).

16. B. J. Spencer and J. Tersoff. Phys. Rev. Lett. 79, 4858 (1997).

17. S. Christiansen, M. Albrecht, H. P. Strunk, H. J. Maier. Appl. Phys. Lett. 64, 3617 (1994).

18. M. Strassburg, V. Kutzer, U. W. Pohl, A. Hoffmann, I. Broser, N. N. Ledentsov, D. Bimberg, A. Rosenauer, U. Fischer, D. Gehrtsen, I. L. Krestnikov, M. Maximov, P. S. Kopev, Zh. I. Alferov. Appl. Phys. Lett. 72, 942 (1998).

19. D. Bimberg, M. Grundmann, N. N. Ledentsov, S. S. Ruvimov, P. Werner, U. Richter, J. Heydenreich, V. M. Ustinov, P. S. Kop'ev, Z. I. Alferov. Thin Solid Films 267, 32 (1995).

20. O. Stier, M. Grundmann, D. Bimberg. Phys. Rev. B 59, 5688 (1999).

21. T. Saito, J. N. Schulman, Y. Arakawa. Phys. Rev. B 57, 13016 (1998).

22. M. Grundmann, O. Stier, D. Bimberg. Phys. Rev. B 52, 11969 (1995).

23. N. Moll, M. Scheffler, E. Pehlke. Phys. Rev. B 58, 4566 (1998).

24. D. Leonard, K. Pond, P. M. Petroff. Phys. Rev. B 50, 11687 (1994).

25. J. M. Moison, F. Houzay, F. Barthe, L. Leprince; E. André, O. Vatel. Appl. Phys. Lett. 64, 196 (1994).

26. Y. Nabetani, T. Ishikawa, S. Noda, A. Sasaki. Appl. Phys. Lett. 76, 3347 (1994).

27. H. Lee, R. Lowe-Webb, W. Yang, P. C. Sercel. Appl. Phys. Lett. 72, 812 (1998).

28. P. B. Joyce, T. J. Krzyzewski, G. R. Bell, B. A. Joyce, T. S. Jones. Phys. Rev. B 58, R15981 (1998).

29. Q-K. Xue, K. Hasegawa, H. Kiyama, T. Sakurai. Jpn. J. Appl. Phys. 38, 500 (1999).

30. M. Schmidbauer, Th. Wiebach, H. Raidt, M. Hanke, R. Köhler, H. Wawra. Phys. Rev. B 58, 10523 (1998).

31. K. Zhang, A. Foede, Th. Schmidt, P. Sonntag, Ch. Heyn, G. Materlik, W. Hansen, J. Falta. Phys. Stat. Sol. (b) 215, 791 (1999).

32. K. Zhang, Ch. Heyn, W. Hansen, Th. Schmidt, J. Falta. Appl. Phys. Lett., in print.

33. S. Song and S. G. J. Mochrie. Phys. Rev. Lett. 73, 995 (1994). 